INVESTIGATION OF ELECTRON LIFETIMES IN MOLYBDENUM WITH DILUTE SUBSTITUTIONAL IMPURITIES*

by

A. J. Arko and F. M. Mueller

\title{
MASTER
}

For Presentation At:

International Conference on Electron Lifetimes in Metals, Eugene, Oregon, July 8-12, 1974 


\section{Investigation of Electron Lifetimes \\ In Molybdenum with Dilute \\ Substitutional Inpuritles*}

by

A. J. Arko and F. M. Muezler.

ARGONNE NATIONAL LABORATORY

9700 South Cass Avenue

Argonne, Illinois 60439

This teport wils prepured as an account of work snunsored $y^{\prime}$ the United States Governmente Nelthes the United States nut the United States Atomic Enercy Commission, nor any of their employees, nor any of their contractors, subcontractors, or their employets, makes any warranty, express or implied, or assumes any legal liability or responsibility for the accuracy, com. pleteness or usefuiness of sas informetion, epparatus, product or process disclused, or represents that its use wauld not infringe privately' owned rights.

To be presented at, and published in the proceedings of, the International Conference on Electron Lifetimes in Metals. Eugene, Oregon, July 8-12, 1974.

*Work supported by the U. S. Atomic Energy Commission. 


$$
\text { by }
$$

A. J. Arko and F. M. Muelier

Argonne National Laboratory Argonne, Illinois

\section{Introduction}

Recently Springford has revlewed ${ }^{1}$ the anisotropy of conduction electron scattering in the noble metals. Since the de Haas-van Alphen effect ylelds a narrow orbital scattering rate, rather than an average over the entire Fermi surface, as is true in resistivity, a systematic set of measurement holds out the promise of providing a detalled set of local scattering rates or lifetimes. Indeed Springford emphastzes this posstbility by denoting such techniques as direct methods.

In this paper we examine the effect of a series of dilute transition and actinfde Impurities on the electronic lifetimes in the host molybdenum. The Fermi surface and electronlc struclure of molybdenum have been studied by a number of workers., 3 Although the specific heat is small, the d-probability at the Fermi energy 18 large, making molyudenum an excellent host to study the effect of transition metal impurities in a transition metal host using the de Haas-van Alphen effect.

In order to make best use of these data it is necessary to invert ${ }^{5}$ to find local or point scattering rates. We have developed ${ }^{6}$ a new and simple scheme for centro-symmetric sheets, which we present in detall in the present paper. We also consider the Important relationships between the errors in the orbital scattering rates and the errors in the derived point scattering rates, since for a constant orbital error, the point error is not constant.

The plan of this paper is as follows: Section II gives the experimental detalls, Section III the Inversion scheme, the results are discussed in Section IV and our conclusions are drawn.

\footnotetext{
* Work performed under the auspices of the U.S. Atomic Energy Comnission.
} 


\section{EXPERIMENTAL}

\section{a) Sample Preparation}

Starting material with a residual resistance ratio of $\approx_{2000}$ was drilled the length of the rod, cleaned, and welded at one end. Impurfties were introduced In the form of wire or chips into the center of the molybdenum rod in the desired concentrations and the combination zone-refined twice. The final concentrations were determined in varfous ways. Tungsten Impurity concentrations were initially obtained to $\pm 10 \%$ using spectrographic analysis. Subsequent concentrations were obtained from resistance ratio measurements. The concentrations of the samples used can be obtalned from Fig. 3. Uranium and ruthenium both have relatively low vapor pressures, thus it was assumed that no material was lost in zone refinIng so that the final concentrations equalled the starting concentrations. The error bars were obtained from the variation of the resistance ratio across the specimen. Because of the high vapor pressure of $\mathrm{Fe}$ at $3000^{\circ} \mathrm{K}$ it was necessary to start with a mixture containing uf to 2 at. \% Te and to make the zone pass at $\approx 10 \mathrm{~mm} / \mathrm{min}$ In order to obtain a single crystal specimen having about 20 P.P.M. Fe. The concentration was determined from a measurement of the susceptibility vs. temperature, determining the Curie constant $\mathrm{C}$ and assuming ${ }^{7} \mathrm{P}_{\text {eff }}=3.0 \mu_{\mathrm{B}}$

The single crystal alloys were sliced into $1 \mathrm{~mm}$ thlck discs and electro-etched to reveal sub-grain boundaries and dislocation etch pits. 8 Samples with final dimensions of $20.5 \mathrm{~mm}$ on a side were cut from sub-grain free regions having the smallest dislocation density. These techniques substantially reduced phase smearing due to inlomogeneities in the specimens. Orbital Dingle temperatures for several equivalent crystallographic (off-axis) directions generally reproduced to better than $0.1^{\circ} \mathrm{K}$.

\section{b) Measurement}

A rotating probe capable of two degrees of freedom was used to orient the sample in the magnetic field. Samples were thus orfented by means of the dH vA effect itself. Problems of backlash are greatly reduced by always rotating or tilting in the same direction; nevertheless, there is a residual uncertainty 
of about $\pm 0.5^{\circ}$ In the orientation. Since one must be oriented ${ }^{9}$ to within * $0.05^{\circ}$ of the axis to avold phase smearing due to sample inhomogeneities one understands the extra precautions needed in sample preparation to reduce such amearing.

Direct measureient by means of a magnetoresistance probe was used to map magnetic field inhomogenelties. 10 It was determined that in the volume occupled by the specimens the fleld inhomogeneity was 3-5 PPM.

The hole octahedron centered at $\mathrm{H}$ was studied in $\mathrm{Mo}(\mathrm{W}), \mathrm{Mo}(\mathrm{Ru})$ and $\mathrm{Mo}(\mathrm{Fe})$ alloys (See Refs, 2 or 3 for the Fermt surface of Mo). Since the octahedron frequency is well above the ellipsold and lens frequencies (the jack frequencies are generally quite weak, appearing only as beats on the octahedren frequency) a single frequency could be selected in the dHvA spectrum and the data could be analyzed without the ald of a computer. In order to measure the Dingle temperature on the remaining sheets of the fermi Surface, an on-line PDP-11/20 mini-computer was ut1lized. Data were taken in equal length blocks between 30 and $65 \mathrm{kG}$ with each block contair.Ing a sufficient number of oscilla. tions to avoid overlapping of peaks in the Fourler transform. A complete Btudy was done on the Mo(U) system only.

The need for accurate effective mass measurements soon became apparent In our work, necessitating the installation of an insert dewar. (Our original data on $M_{0}(\mathrm{~W})$ were taken at $1.9^{\circ} \mathrm{K}$ with no insert dewar.) Masses were measured to within $2 \%$ between $2.0^{\circ} \mathrm{K}$ and $1.1^{\circ} \mathrm{K}$ at fields low enough to avold magnetic Interaction effects. For the ajloy samples studied, this was usually less than $50 \mathrm{kG}$. 


\section{:I. Inversion Scheme}

\section{Forma11s;il}

As has been stressed before ${ }^{6}$ one of the great advantages of measuring wattering probab11'ties by means of the dH vA technique is that for, a suff1: Lently olmple sheet of the Fermi surface, the orbitally averaged scattering ates may be inverted to yleld unlque point scattering rater. The motivation :sr such a procedure, although difficult to actually carry out In practice, :s two-fold: (1) The symmetry and consistency of the data are tested in a savere way; (2) The Inversions are carried out using a model based solely on :ixe experimental symmetry of the data and not, for example, on the assumption :iat the Impurity has a potential which 1 s representable by a particular iorm auffin-tin, for example). This motivation has produced a number of inversion ishemes; the only advantage of ours 1s extreme simplicity.

The basic equation for the orbital Iffetime $\tau_{\text {orb }}$ has been given by Lowndes $\mathbf{g}$

$$
\frac{1}{T_{\text {orb }}}=\frac{1}{T} \int_{0}^{T} \frac{1}{\tau(\vec{k})} \operatorname{dt}(\vec{k})
$$

"ere the period $T=2 \pi / \omega_{c}$, the cyclotron frequency $\omega_{c}=\frac{e H}{m_{c}}$, and $m *$ is the -bLtal mass. Let us define the orbltal Dingle temperature $T_{\text {orb }}=\pi /\left(2 \pi K_{\text {orb }}\right)$ $+f$ the point Dingle temperature $\mathrm{T}_{\mathrm{pt}}(\mathrm{k}) \equiv \mathrm{r} /[2 \pi \mathrm{K} \tau(\vec{k})]$, where $\mathrm{k}$ Is Boltaman const. in (1) becomes

$$
\frac{2 \pi m^{*} c}{e H} T_{o r b}=\int_{0}^{T} T_{p t}(\vec{k}) d t(\underline{\vec{k}}),
$$


where: again, the particular orbital path of $\vec{k}$ is chosen by the left hand side of (1). From the Lorentz relation, $\mathrm{d} \overrightarrow{\mathrm{k}} / \mathrm{dt}=\overrightarrow{\mathrm{evxH}} / \mathrm{hr}$, and converting to path length varlable $d \vec{k}$, we obtain

$$
d t(\vec{k})=\frac{\pi c}{e t} \frac{d \vec{k}}{v_{\perp}}
$$

where $v_{\perp}$ is the component of $\vec{v}$ in the plane normal to $\vec{H}$ at the point $\vec{k}$.

Then (2) becomes:

$$
m *(\hat{\varepsilon}) T_{o r b}(\hat{\varepsilon})=\frac{\pi}{2 \pi} \oint d \vec{k} T_{p t}(\vec{k}) / v_{\perp}(\vec{k}),
$$

where the path of $d \vec{k}$ has been chosen by the magnetic field direction $\vec{H}$, and $\hat{\varepsilon}$ Is a unit vector in the direction of $\vec{H}$. We can gain insight into (4) by the equation for the orbital area ${ }^{11}$

$$
A(\hat{\varepsilon})=\frac{1}{2} \oint \rho^{2}(\hat{\xi}) \delta(\hat{\xi} \cdot \hat{\varepsilon}) \mathrm{d} \Omega(\hat{\xi})
$$

and the related one for the orbital mass $m^{*}(\hat{\varepsilon})$

$$
m * \hat{\varepsilon})=\frac{\pi^{2}}{2 \pi} \oint \frac{p(\hat{\xi}) \Delta(\hat{\varepsilon} \cdot \hat{\xi}) \Omega(\hat{\xi})}{h v_{\perp}^{(\hat{\varepsilon})}}
$$

which is obtained from (5) by the relation $\mathrm{m}^{*}(\hat{\varepsilon})-\left(\hbar^{2} / 2 \pi\right) \mathrm{dA}(\hat{\varepsilon}) / \mathrm{d} \hat{\varepsilon}$. Here we have used $\pi v_{\perp}(\hat{\xi})=(\hat{d}) /(d \vec{p})=d E / d k_{\perp}$, and $\rho(\hat{\xi})$ is the radius vector in the $\hat{E}$ direction. Thus the same ${ }^{11}$ spherical harmonic expansion inverston scheme as was applied to convert areas to radil, may be used to invert orbital Dingle temperatures. The new relations are:

$$
\begin{aligned}
& \mathrm{u}^{*}(\hat{\varepsilon}) \mathrm{T}_{\text {arb }}(\hat{\varepsilon}) \leftrightarrow \frac{\mathrm{t}}{2 \pi} \mathrm{T}_{\mathrm{pt}} \rho(\hat{\xi}) / \mathrm{v}_{\perp}(\hat{\xi}) \\
& \mathrm{m}^{*}(\hat{\varepsilon}) \leftrightarrow \frac{\pi}{2 \pi} \rho(\hat{\xi}) / \mathrm{v}_{\perp}(\hat{\xi})
\end{aligned}
$$


where again the spherical harmonic expansion coefficients of left and right hand sides are related by

$$
a_{\ell}^{m}=c_{\ell}^{m} P_{\ell}(0)
$$

Since $\rho(\hat{\xi}) / \mathrm{y}_{\perp}(\hat{\xi})$ scales out in calculating the ratio of the two right hand sides of (7), It need not be calculated exflicitly.

\section{b) Expansions}

We now take up the relationship of accuracy and the number of useful expansion coefficients. This is particularly important since the derived point Dingle temperature, $\mathrm{T}_{\mathrm{pt}}(\overrightarrow{\mathrm{k}}), \mathrm{hat}$ a variable error as a function of $\vec{k}$. We do not think this has been stressed before.

For simplicity, we will assume that the error in each of the orbital Dingle temperatures $T_{1}$ is uniform $(\overbrace{10 \%})$ although clearly high symmetry points as [100] and [111] have considerably less error than off-axis polnts. The corresponding orbital masses $m_{1}$, have much smaller errors, of the order $1-2 \overline{\%}$, so that these errors can be completely neglected compared to those in $T_{1}$. Thus we need consider only the error in $T_{1}$.

We wish to expand the product $\mathrm{m}_{1} \mathrm{~T}_{1}$ and $\mathrm{m}_{1}$ as:

$$
\begin{aligned}
& m_{1} T_{1}=\sum_{j} s_{1 j} a_{j} \\
& m_{1}=\sum_{j} s_{1 j} b_{j}
\end{aligned}
$$

where the $s_{1 j}$ 's are the spherical (cublc) harmontcs and the $a^{\prime} s$ and $b^{\prime} s$ are the expansion coefficients. By assumption, $m_{i}$ has no error, so the quality of the expansion $\mathrm{J}$ is governed by the $a^{\prime} s$ above. We assume that $a_{j}$ has an ensemble of values, based on the range of each $T_{1}$, with a maximum 11 kelihood ${ }^{12}$ value $a_{j}{ }$. Since 
we expand $m_{1} T_{1}$, we have the product error $\sigma_{1}$ where for the present case

$$
\sigma_{1}=0.10 \mathrm{~m}_{1} \mathrm{~T}_{1} \text {. }
$$

As usual we define a data vector $\vec{X}$ and a measurement matrix $\overleftrightarrow{M}$ by:

$$
\begin{aligned}
& x_{j}=\sum_{i=1}^{N} s_{i j} m_{i} T_{i} / \sigma_{i}^{2} \\
& M_{j l}=\sum_{i=1}^{N} s_{i j} s_{i \ell} / \sigma_{i}{ }^{2}
\end{aligned}
$$

whose least squares (maximum likelihood) solution for the expansion of $m_{1} T_{1}$ is

$$
a_{j} *=\sum_{\ell} M_{j l}^{-1} x_{l}
$$

The standard error in $a_{j}$ squared is

$$
\left(\Delta a_{j}\right)^{2}=\left\langle\left(a_{j}-a_{j} *\right)^{2}\right\rangle=M_{j j}{ }^{-1}
$$

As we discussed above the error in the set of mass coefficlents $b$, will have a smaller error. Let the expansion coefficient of the rhs of ( 7 ) be $c$ and $d$ respectively, related ${ }^{11}$ to $a$ and $b$ by $\left(\begin{array}{l}a \\ b\end{array}\right)=P_{\ell}(0)\left(\begin{array}{l}c \\ d\end{array}\right)$. The standard error in $c$ is in general bigger than that of $a$ and is given by

$$
\left(\Delta c_{j}\right)^{2}=\left(\Delta a_{j}\right)^{2} / P_{j}(0)^{2}
$$

We wish to know the derived exror in $T_{p t}$, the point ingle temperature. We assume that the error in

$$
\pi / 2 \pi \rho(\hat{\xi}) / v_{\perp}(\hat{\xi})=\sum_{j} d_{j} s_{j m}
$$


Is zero. Thus the error is governed by the expansion

$$
z=(\pi / 2 \pi) \rho(\hat{\xi}) T_{p t}(\hat{\xi}) / v_{\perp}(\hat{\xi})=\sum_{j} c_{j} s_{j m}
$$

The variance or mean square deviation is

$$
\begin{aligned}
&\left.\left\langle(z-\bar{z})^{2}\right\rangle=<\left[\sum_{j}\left(c_{j}-\bar{c}_{j}\right) s_{j m}\right]^{2}\right\rangle \\
&\left.\left\langle(z-\bar{z})^{2}\right\rangle=\sum_{j} \sum_{k} \frac{s_{j m}}{P_{j}} \frac{s_{k m}}{P_{k}}<\left(a_{j}-\bar{a}_{j}\right)\left(a_{k}-\bar{a}_{k}\right)\right\rangle \\
&=\sum_{j} \sum_{k}\left(s_{j m} / P_{j}\right)\left(s_{k m} / P_{k}\right) M_{j k}^{-1}
\end{aligned}
$$

So that the error in $f$ is given by the ratio of the square root of (17) divided by the right hand side of (7b).

\section{Results and Discussion:}

A complete set of data for all angles was obtained only on the hole octahedron centered at $H$ (and on:ly in the $M o(W), M o(R u)$ and $M o(F e)$ alloys). Effective masses (m*) were meastu)ed to within $2 \%$ on this sheet and are shown in Fig. 1. The masses of Leaver and Meyers ${ }^{13}$ (used in our original publication ${ }^{5}$ of Mo(W) results) are also shown in the figure. The large disctpeancies are obvious and resulted in erroneous derived Dingle temperatures. We believe that the discrepancies are moet likely due to magnetic interaction effects which become olgnificant in very pure samples and at high fielas. We did not encounter thesc difficulties in our alloy samples but did see them in vecy pure Mo. 
The Dingle temperatures obtained on the hole octahedron are shown in Fig. 2 for the (100) and (110) planes. Any anisotropy that is observed falls for the most part within experimental error. Only in Mo(Ru) do we get an anisotropy which is somewhat larger than the experimental error of $5 \%$ to $10 \%$. But even here the small peaks wear [100] occur in a region where $\mathrm{df} / \mathrm{d} \theta$ is large (f is the frequency and $\theta$ is the angle) thus leaving room for arguemert that in this region we are undarestimating our errors. The $\mathrm{Mo}(\mathrm{Fe})$ and $\mathrm{Mo}(W)$ curves are best fit by a straight line.

F1g. 3 shows a plot of the average $T_{D}$ in each material vs. I/RRR (residual resistance ratio). It can be seen that all the points 1 le very well on one curve whose intercept is less han $0.1^{\circ} \mathrm{K}$. Thus it can be argued that there was little strain in these samples and that phase smearing was relatively salall; i.e., dislocation scattering which does not contribute significantly to the resistivity, afpears to be less than $0.1^{\circ} \mathrm{K}$.

The Mo(Ru) data was inverted, using the above described inversion technique, to obtaln point Dingle Temperatures. Although the orbital temperatures are nearly flat a seemingly large anisotropy can be accidentally obtained in the point IIfetines owing to the large anisotropy in the effective masses. For this reason it is imperative that errors be analyzed carefully.

In Fig. 4 we plot the derived point Dingle temperatures for Mo(Ru) using cubic harmonic analysis. The bars in the figure mark the errors obtained using two expansion coefficients and assuming a constant $10 \%$ error in $\mathrm{T}_{\text {orb }}$. As can be seen, the error in $T_{p t}$ is quite variable being $13.3 \%, 34.0 \%$, and $8.3 \%$ at [110], [111] and [100], re.pectively.

We list in Table I the resulting cubic harmoric coefficients and errors for a serles of low order expansions. As can be seen from the t.able, the error in $\mathrm{T}_{\text {orb }}(10 \%$ compared to the variation of $15 \%$ ) means that the variation in the $n=2$ plot is just significant. The rather large error of 0.076 for $a_{3}$ 
suggests that the $n=3$ expansion is insignificant. Since the rms fitting error for $\mathrm{n}=2$ fitting to $m_{1} \mathrm{~T}_{1}$ is smaller than the assumed experimental error of $10 \%$, the serfes must be cut off at $n=1$; $1 . e .$, the data are equally well fit by a flat line, which lmplies a constant point Dingle temperature of 1.82 degrees.

Thus we conclude that as far as scattering on the octahedron is concerned, the resulting point lifetimes are isotropic to about $5 \%$ in all systems investigated, even though the effective mass varies from 0.75 to 1.22 electrun masses and the Inverse velocity varies from 0.62 to 1.92 . This conclusion is consistent with the constancy of the wave functions found by Myron, Mueller and Freeman ${ }^{4}$ at this conference.

Using the data of Fig. 3, we find that the scattering matrix element per Impurity is $0.685,0.705,0.711$ and $0.094 \mathrm{eV}$ per impurity of $R u, F e$, $U$ and $W$, respectively. Thus in a simple picture the two Impurities Ru and Fe have an excess of two electrons compared to Mo, $U$ has a deficiency of two electrons, and $W$ is homovalent. Although Fe is known to have a moment in Mo, and indeed $\mathrm{Mo}(\mathrm{Fe}$ ) is a Kondo system, 14 appears hat all the scattering from Fe impurities can be accounted for as being due to potential scattering caused by the excess electrons. Any spin-flip scattering is smaller than our experimental error.

The data obtained on other sheets of the Fermi surface in the Mo(U) system are shown in Table II. The Dingle temperatures on the lens are smaller (just outside the range of experimental error) than the octahedron, fack and neck Dingle temperatures, the latter three of which appear to be 1dentical. This too is consistent with the results of Myron ${ }^{4}$ et, aj. who find that all these surfaces are almost purely d-like.

The wave functions on the ellipsoids, however, have considerable p-character and the caluclated point 11fetimes are a factor of two smaller than on the d-like surfaces. Our measured lifetimes appear tu be elther the same or even larger than on the octahedron. It is necessary to point out, however, that 
although our plots of $\log$ (auplitude) vs $1 / \mathrm{H}$ had a very small rms error, nearly all the ellipsoid frequencies are degenerate in the planes measured and we could be on the tall of a long beat if we are not exactly in the plane. Since our $0.5^{\circ}$ error in orlentation is probably sufficient to cause long beats there is some doubt about the numbırs obtained on the ellipsoid.

\section{Acknowledgements}

We vish to thank Climax Molybdenum Co. for spectrographic analysis for the ccncentration of $W$ Impurities, $D r$. D. Lam for the use of his susceptibility equipment to determine the Fe concentration, Dr. L. R. Windmiller for his Fourler transform programs, and C. H. Sowers for technical assistance.

\section{References}

1. M. Springford, Advances in Physics 214931 (1972)

2. T. L. Loucks, Phys. Rev. 139, Al181 (1965

3. D. D. Koelling, F. M. Mueller, J. B. Ketterson and A. J. Arko, (to be published), and references therein.

4. H. W. Myron, F. 3. Mueller, and A. J. Freeman, Paper 69 at this conference,

5. D. H. Lowndes, K. M. Miller, R. G. Poulsen and M. Springford, Proc. Roy Soc. A 331, 497 (1973).

6. A. J. Arko and F. M. Mueller, Phys, Rev. Letters 29,1515 (1972)

7. G. S. Knapp (private communication).

8. D. W. Terwilliger and R. J. Higgins, J. Appl. Phys 43, 3346 (1972).

9. D. W. Terwilliger and R. J. Higgins, Phys. Rev. B 7667 (1973).

10. We thank $C$. H. Sowers for his careful mapping.

11. F. M. Mueller Phys. Rev. 153, 659 (1967).

12. See, for example, the discussion in J. Mathews and R. L. Walker Mathematical Metk ls of Physics (W, A. Benjamin, Inc., N. Y., 1964) p. 365.

13. G. Leaver and A. Myers, Phil. Mag 19, 465 (1969).

14. See, for example, M. P. Maley and R. D. Taylor, Phys. Rev. B 1, 4213 (1970). 
TABLE I

$\begin{array}{cccccccc} & a_{1} & \Delta a_{1} & a_{2} & \Delta a_{2} & a_{3} & \Delta a_{3} & \text { Rms } \\ 1 & 5.528 & 0.106 & 0 & 0 & 0 & 0 & 23.6 \\ 2 & 5.801 & 0.110 & 0.883 & 0.096 & 0 & 0 & 5.85 \\ 3 & 5.792 & 0.111 & 0.895 & 0.096 & 0.053 & 0.076 & 4.55\end{array}$

${ }^{*} \operatorname{Rms} 13$ in $\%$ and $=$ mean error $/\left\langle m_{1} T_{1}\right\rangle$ 
TABLE II

Dingle Temperatures for Mo(U)

\begin{tabular}{|l|l|l|l|} 
Sheet & $\begin{array}{l}\text { Frequency } \\
\times 10^{6} \text { Gauss }\end{array}$ & Orientation & $T_{\text {orb }}$ \\
\hline Octahedron & 103 & {$[111]$} & $1.34 \pm 0.05$ \\
\hline Octahedron & 151 & {$[100]$} & $1.28 \pm 0.05$ \\
\hline Jack & 85 & {$[111]$} & $1.32 \pm 0.05$ \\
\hline Lens & 5.2 & {$[100]$} & $1.12 \pm 0.1$ \\
\hline Lens & 8.2 & {$[100]$} & $1.14 \pm 0.1$ \\
\hline Neck & 13 & $\begin{array}{l}10^{\circ} \text { from } \\
{[100]}\end{array}$ & $1.3 \pm 0.1$ \\
\hline El1ipsoid* & 23 & {$[100]$} & 1.26 \\
\hline E11ipsoid* & 28 & $\begin{array}{l}15^{\circ} \text { from } \\
{[100]}\end{array}$ & 2.56 \\
\hline
\end{tabular}

* Error not known 


\section{FIGURE CAPTIONS}

Fig. 1. Effective masses on the hole octahedron of the Fermi surface of tho In (100) and (110) planes. The dached curve are the data of Leaver and Meyers ${ }^{13}$. The masses with value $\approx 1.0$ near [111] are for the electron jack.

Fig. 2. Measured orbital Dingle temperatures on the hole octahedron In the (100) and (110) planes for three substitutional impurities in Mo.

F18. 3. Plot of average orbital Dingle temperature vs. Inverse residual resistance ratio in the samples measured. The small intercept Indicates small dislocation scattering.

F1g. 4. PoInt Dingle temperatures obtained by inverting the Mo(Ru) data of FIg. 2 . 


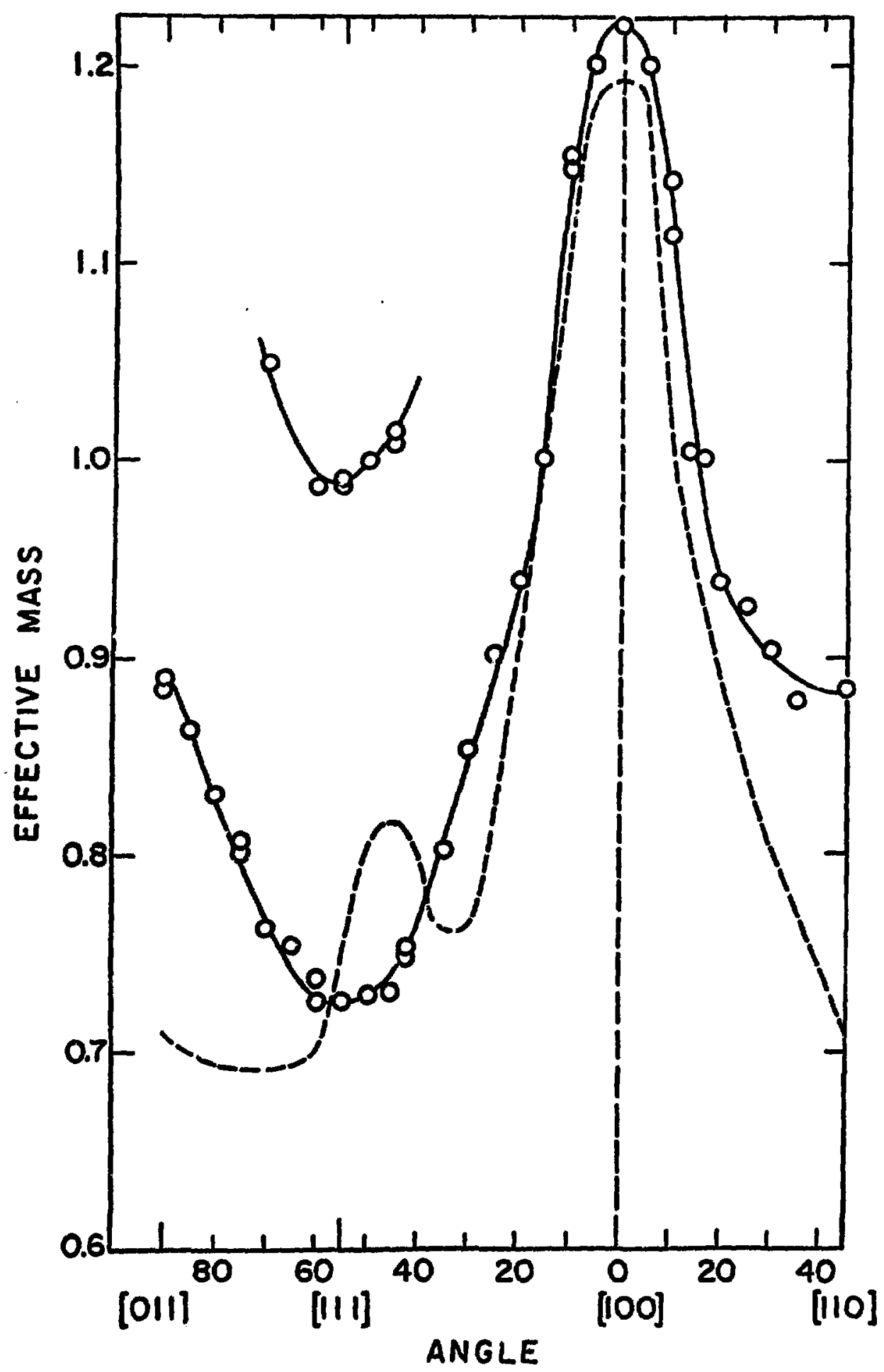




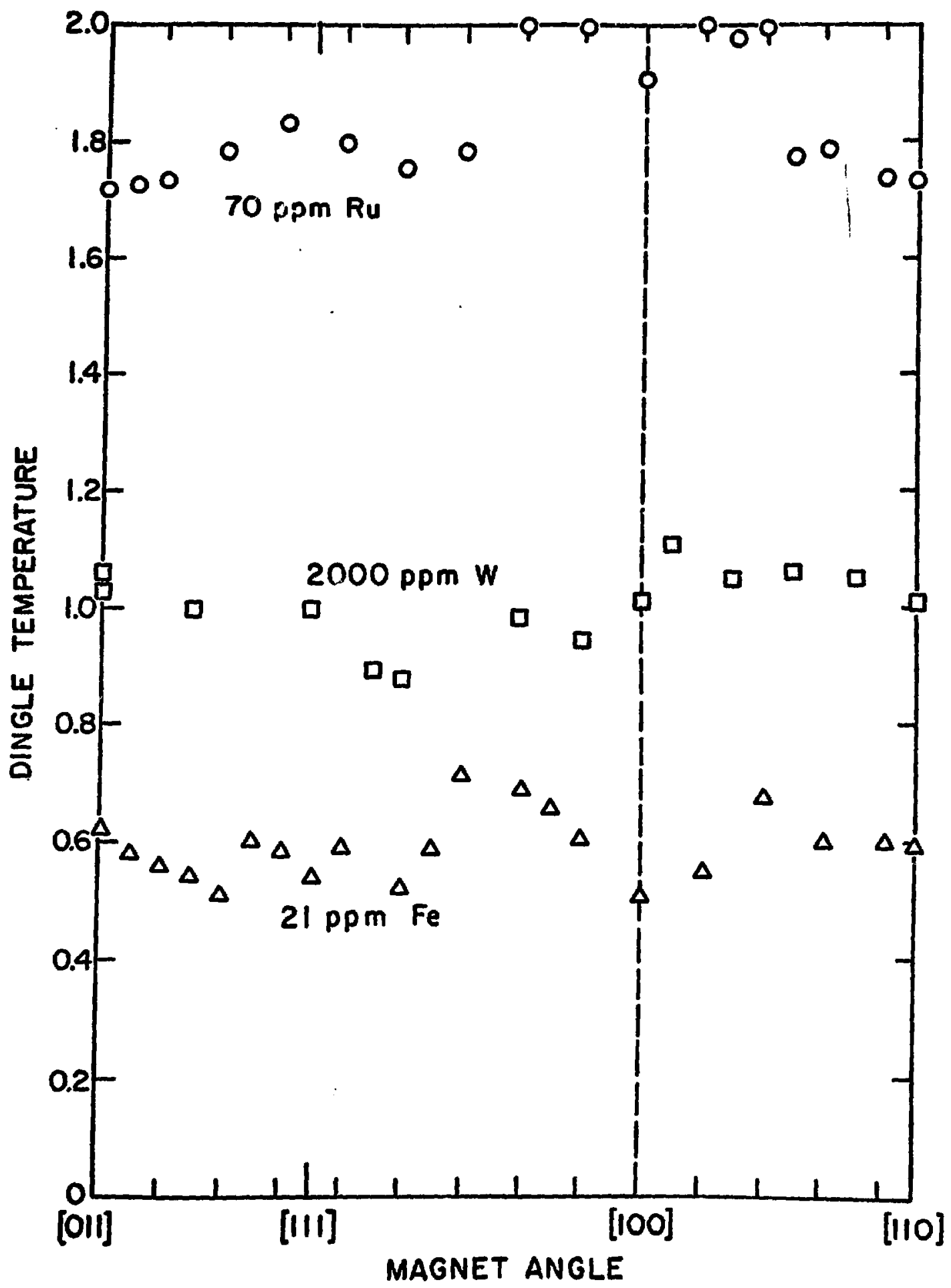




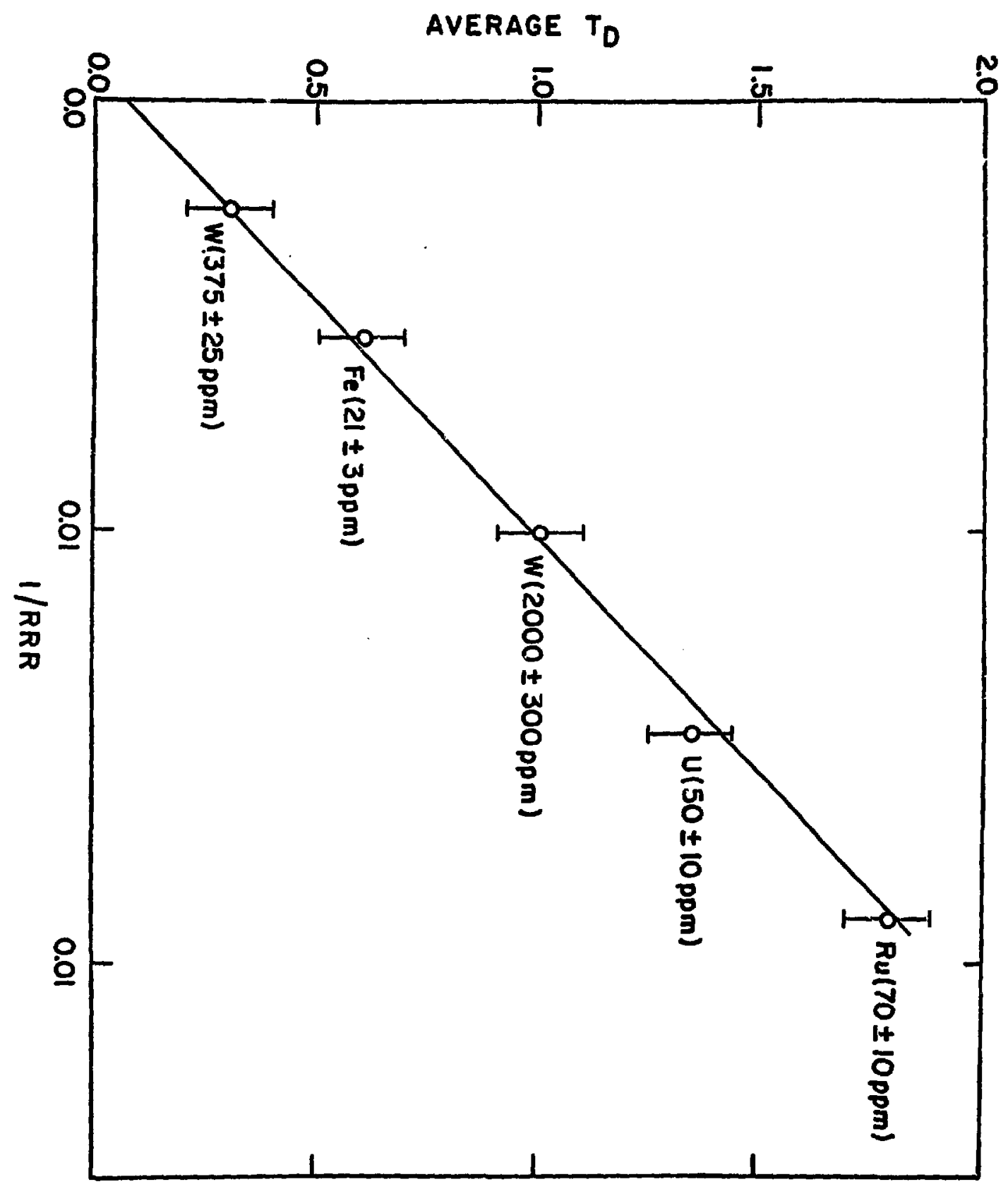




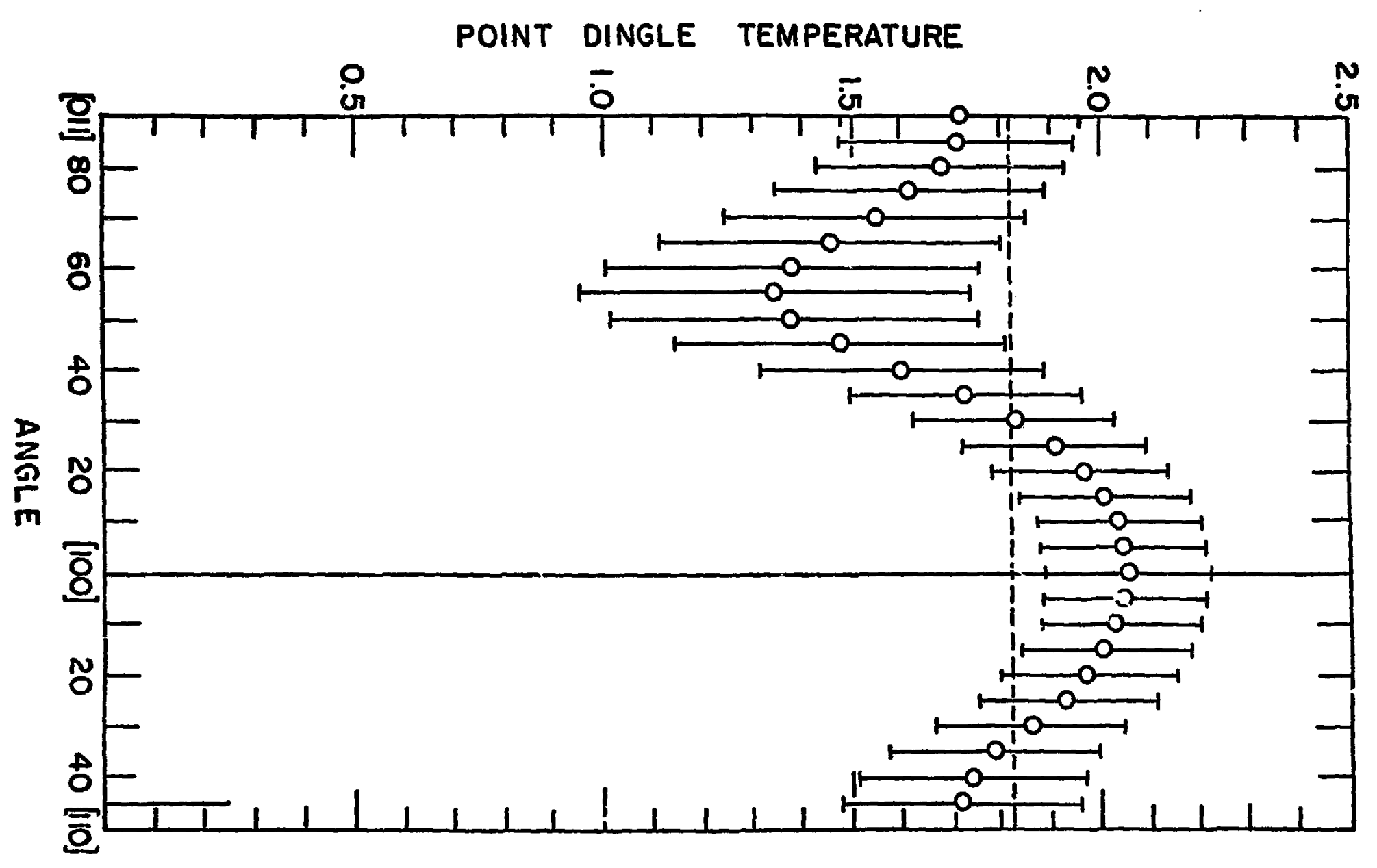

